Supporting Information for:

\title{
Coupled Double Optical Stark Effect in CdSe Colloidal Nanoplatelets
}

Dongmei Xiang, ${ }^{1 \dagger}$ Yulu Li, ${ }^{1 \dagger}$ Lifeng Wang, ${ }^{1,2}$ Yang Zhao, ${ }^{3}$ and Kaifeng $\mathrm{Wu}^{1 *}$

${ }^{1}$ State Key Laboratory of Molecular Reaction Dynamics and Dynamics Research

Center for Energy and Environmental Materials, Dalian Institute of Chemical

Physics, Chinese Academy of Sciences, Dalian, Liaoning 116023, China

${ }^{2}$ University of the Chinese Academy of Sciences, Beijing 100049, China

${ }^{3}$ Dalian National Laboratory for Clean Energy, Dalian Institute of Chemical Physics,

Chinese Academy of Sciences, Dalian, Liaoning 116023, China

*Address correspondence to: kwu@dicp.ac.cn 



b $\sigma^{+}$pump/ $\sigma^{-}$probe

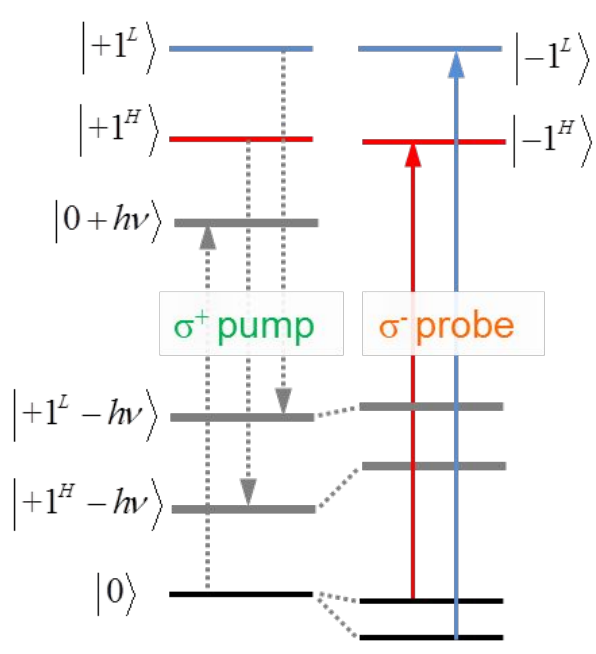

Figure S1. Excitonic representation of the double-OSE. $\mid 0>$ is the ground state and $\mid+(-) 1^{H>}$ and $\mid+(-) 1^{L>}$ are the $\mathrm{HH}$ and $\mathrm{LH}$ excitons, respectively, with angular momentum projections of $+(-) 1$. (a) Assuming that the pump photon is a sub-gap $\sigma^{+}$ photon, the photon-dressed states of the three-level system are $|0+h v>|+,1^{H}-h v>$ and $\mid+1^{L}-h v>$. Repulsion between $\mid 0+h v>$ and $\mid+1^{H}>$ or $\mid+1^{L}>$ upshifts the latter; the shift is stronger for $\mid+1^{H>}$ than $\mid+1^{L>}$ because of the smaller detuning energy and higher oscillator strength of $\mid+1^{H>}$ than $\mid+1^{L}>$. Similarly, repulsion between $\left|+1^{H_{-}} h v\right\rangle$ $\left(\mid+1^{L_{-}} h v>\right)$ and $\mid 0>$ downshifts the latter and the shift induced by $\mid+1^{H_{-}}-h v>$ is stronger than $\mid+1^{L}-h v>$. As a result, when probed with $\sigma^{+}$photons, both $\mid 0>$ to $\mid+1^{H}>$ and $\mid 0>$ to $1+1^{L_{>}}$transitions are blue-shifted. (b) More exotic behaviors emerge when the probed photons are $\sigma$, i.e., probing the $\mid 0>$ to $\mid-1^{H}>$ and $\mid 0>$ to $\mid-1^{L}>$ transitions. The $\mid-1^{H}>$ and $\mid-1^{L}>$ states are not shifted as they do not interact with the $\mid 0+h v>$ photon-dressed state due to their different helicities. However, the $\mid 0>$ state is still down-shifted due to its repulsion with $\mid+1^{H}-h v>\left(\mid+1^{L}-h v>\right)$. Consequently, $\mid 0>$ to $\mid-1^{H}>$ and $\mid 0>$ to $\mid-1^{L>}$ transitions will be blue-shifted. 



Figure S2. Double-OSE at high pump intensity. (a,b) 2D pseudo-color plots of the TA spectra of CdSe NPLs dispersed in hexane measured with (a) co- $\left(\sigma^{+}, \sigma^{+}\right)$and (b) counter-circularly $\left(\sigma^{-}, \sigma^{+}\right)$polarized pump/probe pulses. The pump wavelength is 548 $\mathrm{nm}(2.26 \mathrm{eV})$ and the intensity is $0.35 \mathrm{GW} / \mathrm{cm}^{2}$. (c,d) Temporal profiles of the (c) HH and (d) LH bleach features measured with co- (orange) and counter-circularly (green) polarized pump/probe pulses. Weak state-filling signals arising from two-photon absorption can be observed, but their contribution at time zero is negligible. 


\section{Experimental Methods}

Synthesis of CdSe NPLs. We synthesized CdSe NPLs by modifying previously reported procedures. ${ }^{1}$ Details were recently reported elsewhere. ${ }^{2}$

Pump-probe experiment. The femtosecond pump-probe TA measurements were performed using a regenerative amplified Ti:sapphire laser system (Coherent; $800 \mathrm{~nm}$, $6 \mathrm{~mJ}$ per pulse, and $1 \mathrm{kHz}$ repetition rate) as the laser source and a Femto-100 spectrometer (TimeTech LLC) as the spectrometer. Details can be found in our prior reports..$^{3,4}$

\section{Fitting absorption spectrum}

We fit the absorption spectrum of CdSe NPLs using a previously-established QW absorption model. 5 The excitonic absorption of a QW exciton is:

$$
\alpha_{X}(E)=\frac{1}{2 \eta}\left[\operatorname{erf}\left(\frac{E-E_{0}}{\gamma_{X}}-\frac{\gamma_{X}}{2 \eta}\right)+1\right] \cdot \exp \left(\frac{\gamma_{X}{ }^{2}}{4 \eta^{2}}-\frac{E-E_{0}}{\eta}\right)
$$

where $E_{0}$ is the absolute energy of the exciton, $\gamma_{X}$ is the line width and $\eta$ is an asymmetric broadening factor. The corresponding absorption of the continous band is:

$$
\alpha_{C}(E)=\frac{H_{C}}{2}\left[\operatorname{erf}\left(\frac{E-E_{0}-E_{b}}{\gamma_{C}}\right)+1\right]
$$

where $H_{C}$ is the step height of the contiuum edge, $\gamma_{C}$ is itswidth and $E_{b}$ is the exciton binding energy. The absorption of CdSe NPLs is the sum of excitonic and continuous absorptions of $\mathrm{HH}, \mathrm{LH}$ and $\mathrm{SO}$ bands: 


$$
\alpha(E)=\sum_{i=H H, L H, S O} A_{i}\left(\alpha_{X, i}(E)+\alpha_{C, i}(E)\right)
$$

The fitting parameters are listed in Table S1.

Table S1. Fitting parameters for the NPL absorption spectrum

\begin{tabular}{lrrr}
\hline & \multicolumn{1}{l}{ HH } & \multicolumn{1}{l}{ LH } & \multicolumn{1}{l}{ SO } \\
\hline $\boldsymbol{E}_{\boldsymbol{b}}(\mathrm{eV})$ & 0.13 & 0.29 & 0.2 \\
$\boldsymbol{E}_{\boldsymbol{0}}(\mathrm{eV})$ & 2.41 & 2.56 & 2.9 \\
$\boldsymbol{\gamma}_{\boldsymbol{X}}(\mathrm{eV})$ & 0.013 & 0.047 & 0.16 \\
$\boldsymbol{H}_{\boldsymbol{C}}(\mathrm{eV})$ & 4.8 & 4.02 & 0.6 \\
$\boldsymbol{\gamma}_{\boldsymbol{C}}(\mathrm{eV})$ & 0.08 & 0.06 & 0.08 \\
$\boldsymbol{\eta}$ & 0.056 & 0.038 & 0.06 \\
$\boldsymbol{A}$ & 0.08 & 0.045 & 0.006 \\
\hline
\end{tabular}

\section{Calculating the pulse intensity}

The pulse intensity is the ratio of average pulse energy density to the pulse duration. The diameter of the pump beam was measured using a knife-edge method and was estimated as 930 and $850 \mu \mathrm{m}$ for the 548 and $557 \mathrm{~nm}$ pump beams, respectively. The pulse duration was taken as the response the full width at half maximum of TA instrument response function (IRF), which was $\sim 170$ fs. Using these parameters, the pump pulse intensities were calculated.

\section{Calcualting the spectral weight transfer (SWT) and OSE shift ( $\delta E)$}

For an absorption spectrum of an aribitury shape deseribed by a function $A(E)$, where $E$ is the energy and $E_{0}$ is the absorption peak, the transient change of the spectrum due to a small positive shift of $\delta E$ can be approximated by the first derivative of the function and $\delta E$ :

$$
\delta A(E)=A(E-\delta E)-A(E) \approx-\frac{d A(E)}{d E} \delta E
$$

Thus, for an analytical function $A(E), \delta E$ can be easily estimated using the first derivative of $A(E)$. In the case of an unknown function, however, $\delta E$ should be estimated through the so-called spectral weight transfer (SWT) approach:7,8 


$$
\mathrm{SWT}=\int_{0}^{E_{0}} \delta A(E) d E
$$

Combining eqs. S4 and S5, the SWT can be derived as:

$$
\mathrm{SWT}=-\delta E \int_{0}^{E_{0}} \frac{d A(E)}{d E} d E=-\delta E\left(A\left(E_{0}\right)-A(0)\right) \quad(\mathrm{S} 6) .
$$

Thus, the energy shift $\delta E$ due to the OSE can be calculated as:

$$
\delta E=-\frac{\mathrm{SWT}}{A\left(E_{0}\right)}
$$

In our experiment, it is important to note that the absorption $A\left(E_{0}\right)$ of the excitons, particularly for the LH exciton, cannot be directly read out from the absorption spectrum, as there are many absorptions overlapped with each other. By fitting the absorption spectrum of the solution sample using the method described above, we found that the $\mathrm{HH}$ and $\mathrm{LH}$ exciton absorptions (OD) are 0.375 and 0.162 , respectively.

\section{Fitting the OSE shift and calculating the transition dipole}

In principle, with the ratio of $\mu_{H H}^{2}: \mu_{L H}^{2}$ ratio of $\sim 1.8: 1$, we can simultaneously fit the power-dependent OSE shifts in Fig. 4d in the main text. However, we find such a fit to be insufficient. A practical reason is the slight imperfect optical orientation due to the imperfect circular polarizations of the pump/probe pulses and/or random orientation of NPLs in solution. In order to account for this effect, we phenomenologically introduce a factor $f$. Using this factor, the experimental OSE shifts ( $\delta E^{c o, \exp }$ and $\delta E^{c o u n t e r, \text { exp }}$ ) are expressed as liner combinations of the ideal $\delta E^{c o}$ and $\delta E^{\text {counter }}$ :

$$
\begin{aligned}
& \delta E^{c o, \exp }=f \cdot \delta E^{c o}+(1-f) \cdot \delta E^{\text {counter }} \\
& \delta E^{\text {counter }, \exp }=f \cdot \delta E^{\text {counter }}+(1-f) \cdot \delta E^{c o}
\end{aligned}
$$


For example, the expression for $\delta E_{H H}^{c o, \exp }$ is:

$$
\delta E_{H H}^{c, \text { exp }}=f \cdot \mu_{H H}^{2}\left\langle E^{2}\right\rangle / \Delta_{H H}+(1-f) \cdot \mu_{L H}^{2}\left\langle E^{2}\right\rangle / 2 \Delta_{L H}=\mu_{H H}^{2}\left\langle E^{2}\right\rangle\left[f / \Delta_{H H}+(1-f) / 3.6 \Delta_{L H}\right]
$$

The other expressions can be similarly derived. $\left\langle E^{2}\right\rangle$ is the time-averaged value of the electric field squared that can be calculated from the pump intensity $I_{0}$ through:

$$
\left\langle E^{2}\right\rangle=I_{0} /\left(\varepsilon_{0} \sqrt{\varepsilon_{s}} c\right)
$$

where $\varepsilon_{s}$ is the effective dielectric constant of the NPL/solvent system, $\varepsilon_{0}$ is the vacuum permittivity, $c$ is the light velocity. Following a recent study on a similar system, ${ }^{9}$ we use $\varepsilon_{s}=1.8$. The choice of $\varepsilon_{s}$ only affects the absolute value of $\mu_{H H}$ calculated at the end, but not the fit of the OSE shift. With the equations defined above, we can fit the data in Fig. $4 \mathrm{~d}$ using $f=0.80$ and $\mu_{H H}=28.6 \mathrm{D}$.

\section{References for SI:}

(1) Ithurria, S.; Tessier, M. D.; Mahler, B.; Lobo, R. P. S. M.; Dubertret, B.; Efros, A. L. Colloidal Nanoplatelets with Two-Dimensional Electronic Structure. Nat. Mater. 2011, 10, 936-941.

(2) Xiang, D.; Li, Y.; Wang, L.; Ding, T.; Wang, J.; Wu, K. Electron and Hole Spin Relaxation in CdSe Colloidal Nanoplatelets. J. Phys. Chem. Lett. 2020, 12, 86-93.

(3) Li, Y.; Luo, X.; Liu, Y.; Lu, X.; Wu, K. Size- and Composition-Dependent Exciton Spin Relaxation in Lead Halide Perovskite Quantum Dots. ACS Energy Lett. 2020, 5, 1701-1708.

(4) Li, Y.; He, S.; Luo, X.; Lu, X.; Wu, K. Strong Spin-Selective Optical Stark Effect in Lead Halide Perovskite Quantum Dots. J. Phys. Chem. Lett. 2020, 3594-3600.

(5) Leosson, K.; Jensen, J. R.; Langbein, W.; Hvam, J. M. Exciton localization and interface roughness in growth-interrupted GaAs/AlAs quantum wells. Phys. Rev. B 2000, 61, 10322-10329.

(6) Grim, J. Q.; Christodoulou, S.; Di Stasio, F.; Krahne, R.; Cingolani, R.; Manna, L.; Moreels, I. Continuous-wave biexciton lasing at room temperature using solution-processed quantum wells. Nat. Nanotechnol. 2014, 9, 891-895. 
(7) Giovanni, D.; Chong, W. K.; Dewi, H. A.; Thirumal, K.; Neogi, I.; Ramesh, R.; Mhaisalkar, S.; Mathews, N.; Sum, T. C. Tunable room-temperature spin-selective optical Stark effect in solution-processed layered halide perovskites. Sci. Adv. 2016, 2, e1600477.

(8) Sie, E. J.; McIver, J. W.; Lee, Y.-H.; Fu, L.; Kong, J.; Gedik, N. Valley-selective optical Stark effect in monolayer WS2. Nat. Mater. 2014, 14, 290.

(9) Diroll, B. T. Circularly Polarized Optical Stark Effect in CdSe Colloidal Quantum Wells. Nano Lett. 2020, 20, 7889-7895. 